\title{
CO-REGISTRATION OF TLS POINT CLOUDS WITH SCAN-PATCHES AND BIM-FACES
}

\author{
F. Gruner ${ }^{1}$, E. Romanschek ${ }^{1}$, D. Wujanz ${ }^{2}$, C. Clemen ${ }^{1}$ \\ ${ }^{1}$ HTW Dresden - University of Applied Sciences, Faculty of Spatial Information, Dresden, Germany \\ \{felix.gruner2, enrico.romanschek, christian.clemen\}@htw-dresden.de \\ 2 technet GmbH, Am Lehnshof 8, 13467 Berlin, Germany \\ daniel.wujanz@technet-gmbh.com
}

Commission V, WG V/7

KEY WORDS: Co-Registration, BIM, point cloud, TLS.

\begin{abstract}
:
To geometrically compare point clouds with Building Information Modeling (BIM) models, they must be transformed to the same coordinate system. The determination of the transformation parameters is called registration. If another model is used instead of control points, the term co-registration has recently been established. The plane-based (relative) registration of point clouds from terrestrial laser scanning (TLS) is successfully used in practice with high accuracy and quality assurance. Based on this, we present a special co-registration approach, that makes use of planes from BIM models. Most buildings have a large number of planar faces, so it is obvious to use faces from BIM. We present intermediate research results of an approach that uses the local planes (patches) of the TLS point clouds also to determine the transformation parameters for the registration to BIM. The aim is to avoid the need of ground control points. The approach requires a (partially) existing BIM model in which the highly precise point cloud is to be registered. In the long term, the procedure is developed for automated construction progress documentation.
\end{abstract}

\section{INTRODUCTION}

\subsection{Background}

Static terrestrial laser scanners (TLS) measure a point cloud which is given in a local scanner coordinate system. These individual point clouds must be transformed into a common coordinate system. There are various methods for this. On the one hand, there is point-cloud to point-cloud registration. Here, overlapping areas of the data sets are iteratively adjusted using the Iterative Closest Point (ICP) algorithm (Besl and McKay, 1992). For this algorithm to be successful, a good preorientation is necessary. For high precision engineering surveys, artificial target marks, i.e. checkerboard marks or reference spheres are used for registration. This procedure is currently the most frequently used. However, the prerequisite for this is the placement of survey markers and an engineering surveying, which requires additional equipment (e.g. total station), time and costs.

A complementary or even substituting approach is possible with natural primitives on the surveyed object. In general, this approach detects diverse types of geometric primitives from the point clouds, such as corners, lines, planes, cylinders or spheres, and compares their position to each other to create a link. The natural targets supplement or replace the marked fixed points and can be automatically extracted from the given point clouds. The use of natural targets alone would lead to a relative registration. In order to achieve a absolute registration it is usually necessary to determine six transformation parameters. This means that the entire point cloud is transformed into a superordinate coordinate reference system, just by a few and well placed control points.

For our research we use the software Scantra developed by technet $\mathrm{GmbH}^{1}$. Scantra is used for the automatic registration of

\footnotetext{
1 https://www.technet-gmbh.com/en/products/scantra/
}

laser scan point clouds. The software enables fast relative registration without the use of targets, but via natural planes that are automatically detected in the scans. Scans of different file formats can be processed in the registration. By this, we utilize the very fast calculation and software framework for our research.

This paper describes the first results of our research, that introduce BIM planes to the registration with Scantra, aiming to offer a co-registration between laser scans and a BIM model.

\subsection{Problem Statement}

The following four research questions are answered throughout this contribution:

- What are the benefits for using faces/planes instead of control points?

- How is the engineering coordinate reference system defined with planes?

- Which building components (BIM) suit the novel approach?

- Which accuracy is achieved for the co-registration and is it documented?

\subsection{Structure of the paper}

The paper has the following structure: This section gives a short introduction to the topic. In Section 2 the relevant related academic work is shortly presented. The main part of this paper is Section 3, which describes our research approach in detail. In Section 4 an assessment of the quality of the achieved result follows. The paper cloeses with Section 5 as conclusion and outlook. 


\section{RELATED WORK}

The intermediate research results, presented in this paper are industry driven. However, they are based on academic work, that was published in (Gielsdorf et al., 2004) and (Wujanz et al., 2018).

The goal of markerless registration was already addressed in the early days of laser scanning, for example at (Böhm and Becker, 2007). The connection of $3 \mathrm{D}$ building models and point clouds has been addressed for more than ten years, e.g. by (Bosché, 2010). Here the point cloud was registered on a CAD model using the ICP algorithm. The shortest distance to the model surface was calculated for the target vs. actual comparison. A little later, (Zhang and Arditi, 2013) developed a program that also investigates the intersection of the point cloud and CAD object. It is checked whether the construction object is installed or whether the point cloud does not represent the object. A comprehensive overview on referencing methodologies is given by (Wujanz, 2016).

On the subject of co-registration point clouds with BIM, (Martens and Blankenbach, 2020) have published a voxel-based approach and recently (Kaiser et al., 2022) a method for coregistration of photogrammetric image blocks and BIM faces.

\section{CO-REGISTRATION WORKFLOW}

In this section we describe the general procedure (see Figure 1) and individual sub-steps. It has to be noted that the steps described in subsection 3.1 and subsection 3.3 are necessary, but are not the subject of research, but commercial software.

\subsection{Laser scan registration}

The block adjustment is prepared by numerous, mostly automated, work steps: After the scans have been loaded to the software, they are connected with edges to create an observationtopology (Figure 2). The connections indicate that two scans can be linked across identical planes, which means, they cover an overlapping visual area. These connections can also be determined automatically in Scantra to a large extent. This is done via corner matching. After the observation-topology has been derived, the plane matching can be carried out semiautomatically for each connecting line. The result of the plane matching is a set of patch-patch pairs for each edge in the observation-topology.

If the overlapping areas between two scans are large enough, this is done automatically. Otherwise, three pairs of planes must be initially matched by manual clicking. Then the remaining combinations are found automatically. When all the connections have been processed, the quality of the individual plane matches is displayed as shown in Figure 2. Each of the small circles symbolizes a scan station. Red lines mean that the standard deviation of the translation is greater than 3 millimeters, green if it is better than 1 millimeter and yellow if it is in between. Otherwise, three pairs of planes must be initially matched by manual clicking. Then the remaining combinations are found automatically. When all the connections have been processed, the quality of the individual plane matches is displayed as shown in Figure 2. Each of the small circles symbolizes a scan station. Red lines mean that the standard deviation of the translation is greater than 3 millimeters, green if it is better than 1 millimeter and yellow if it is in between.

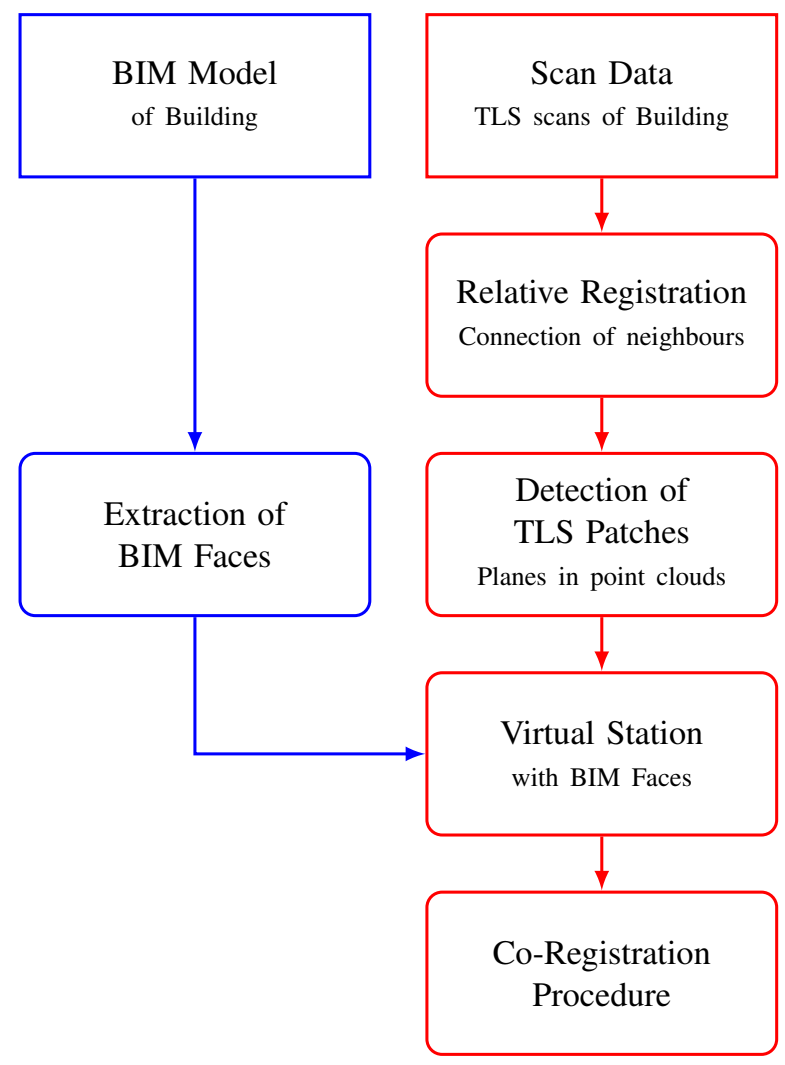

Figure 1. The co-registration starts with the local scan data (red) and the BIM model (blue). Several pre-processing steps are required to finally co-register the point cloud in the BIM model.

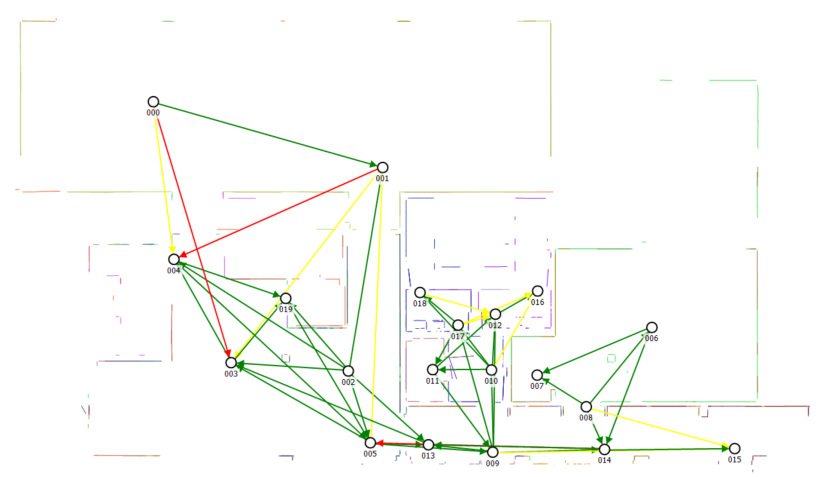

Figure 2. Observation-Topology and connections of the scans in Scantra with cross-section of the point cloud in the background

Red lines must be checked to exclude gross errors. For example, reflections in glass or in long corridors can lead to incorrect connections. A good network configuration is also important to achieve redundancy and stability. A block adjustment optimizes the relative location and orientation of all laser scans based on redundantly captured areas. After that, all relative transformation parameters are adjusted and controlled. This allows the scans to be transformed into the system from one (any) station. In current engineering projects, the transformation to the subordinate engineering datum (absolute registration) can now be established with just a few pre-surveyed control points.

\subsection{Extraction of BIM Faces}

The novelty of our approach is to introduce BIM faces as TLSpatches to the procedure described in the previous subsec- 
tion 3.1. So to speak: From the perspective of the registration software, the digital building model (BIM) becomes nothing more than a TLS standpoint. The term virtual station is used to illustrate that perspective.

In (non-infrastructure) buildings, many construction components are right-angled, parallel or aligned. For this reason, only a few surfaces of the components need to be selected and their faces are extracted from BIM.

During the extraction, each BIM face receives an unique identifier, a 3D-point (on the face) and a normal vector. The unique identifier is required, for example, in later steps and to visualize calculation results in the BIM software. In our current user interface just a few planes must be named uniquely in order to be identifiable in the registration software. All other faces are named programmatically. In Figure 3 tinted planes have been arbitrary named according to their color as also displayed in Figure 6. It is important that at least three approximately right-angled BIM faces are selected, in order to avoid a rank deficiency in the least squares adjustment of the registration.

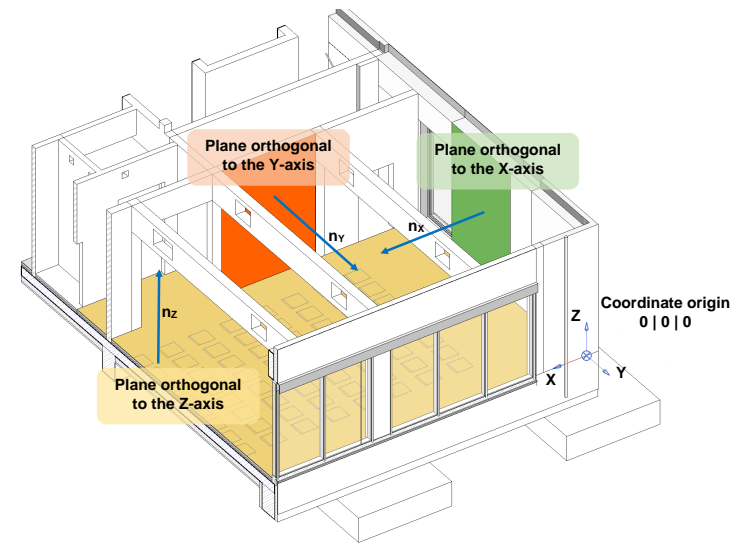

Figure 3. Requirement of the BIM faces for co-registration: Orthogonal position to one of the 3 coordinate axes

In the right part of Figure 3, the BIM coordinate origin is visible. The BIM coordinate system is aligned to the intersection of the wall axes. With our current implementation, the building axes must be parallel to the coordinate axes, but this is most often the case and common for new buildings. Pleas note: The aim of our approach is to transform the TLS point cloud into this coordinate system.

\subsection{Detection of TLS Patches}

The plane detection procedure in Scantra is described in detail in (Wujanz et al., 2018, Section 2.1) and is only summarized in this paper. In contrast to other methods, the plane detection in Scantra is based on a reduction to a two-dimensional problem. For this purpose, an intensity image (Figure 4) is first created from the unstructured point cloud.

The pixel coordinates $p_{x}$ and $p_{y}$ of a scanned point can be calculated with

$$
p_{x}=\operatorname{round}\left(\left(\theta_{i}-\theta_{\min }\right) / \Delta \theta\right)
$$

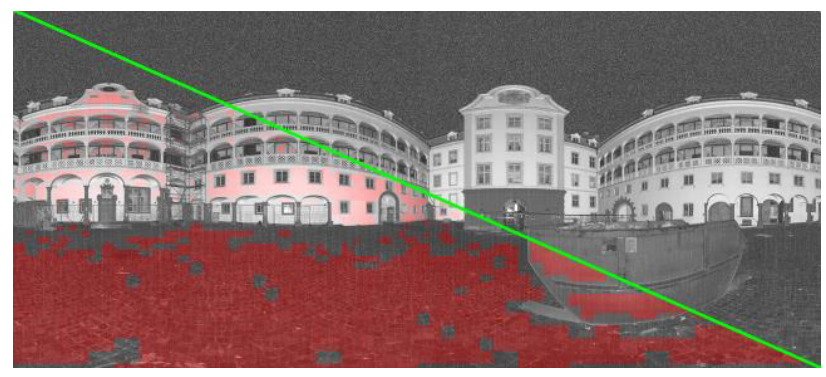

Figure 4. Generated intensity image (upper right) and detected planes tinted in red (lower left) (Wujanz et al., 2018)

$$
p_{y}=\operatorname{round}\left(\left(\phi_{i}-\phi_{\min }\right) / \Delta \phi\right)
$$

Here $\theta_{i}$ and $\phi_{i}$ are the polar components of a measured point (which are calculated from the Cartesian coordinates) and $\theta_{\text {min }}$ and $\phi_{\min }$ are the minimum values of all points. $\Delta \theta$ and $\Delta \phi$ are the defined (scan file header) vertical respectively horizontal angular resolutions of the scan. Using Equation 1 and equation Equation 2 as well as the intensity values of the scanned points, a grayscale image in the form of a two-dimensional matrix can now be created.

The entire data set in the form of the created matrix is then divided into subsets of equal size using a quadtree beased index structure. All points (observations) of a single patch should lie on the same geometric plane. With the following simple observation equation

$$
v_{i}=n_{x} x_{i}+n_{y} y_{i}+n_{z} z_{i}-d
$$

the plane parameters for each patch are adjusted. Here $n_{x}, n_{y}, n_{z}$ are the components of the plane normal, $d$ is the distance of the plane to the origin and $x_{i}, y_{i}, z_{i}$ are the threedimensional coordinates of the observation. If any of the observations of the subset fails the model test, the current set is divided into four subsets of equal size. The procedure is repeated until all observations (TLS points) within a subset pass the stochastic model test.

At the end of the procedure, all planes of a scan with their individual stochastic properties (variance-covariance-matrix) have been determined and the original points of the scan are no longer required. In Figure 4 an intensity image with all detected planes can be seen.

The detection of patches can be parametrized, depending on engineering use-case, scanner hardware, object surfaces or distance, e.g. by the following parameters:

- Maximum points to be read per scan (default value: 20.000.000)

- Maximum number of planes per scan (default value: 1.200)

- Limit for planarity (default value: $0.005 \mathrm{~m}$ )

- Minimum number of scan points per patch (default value: 50) 


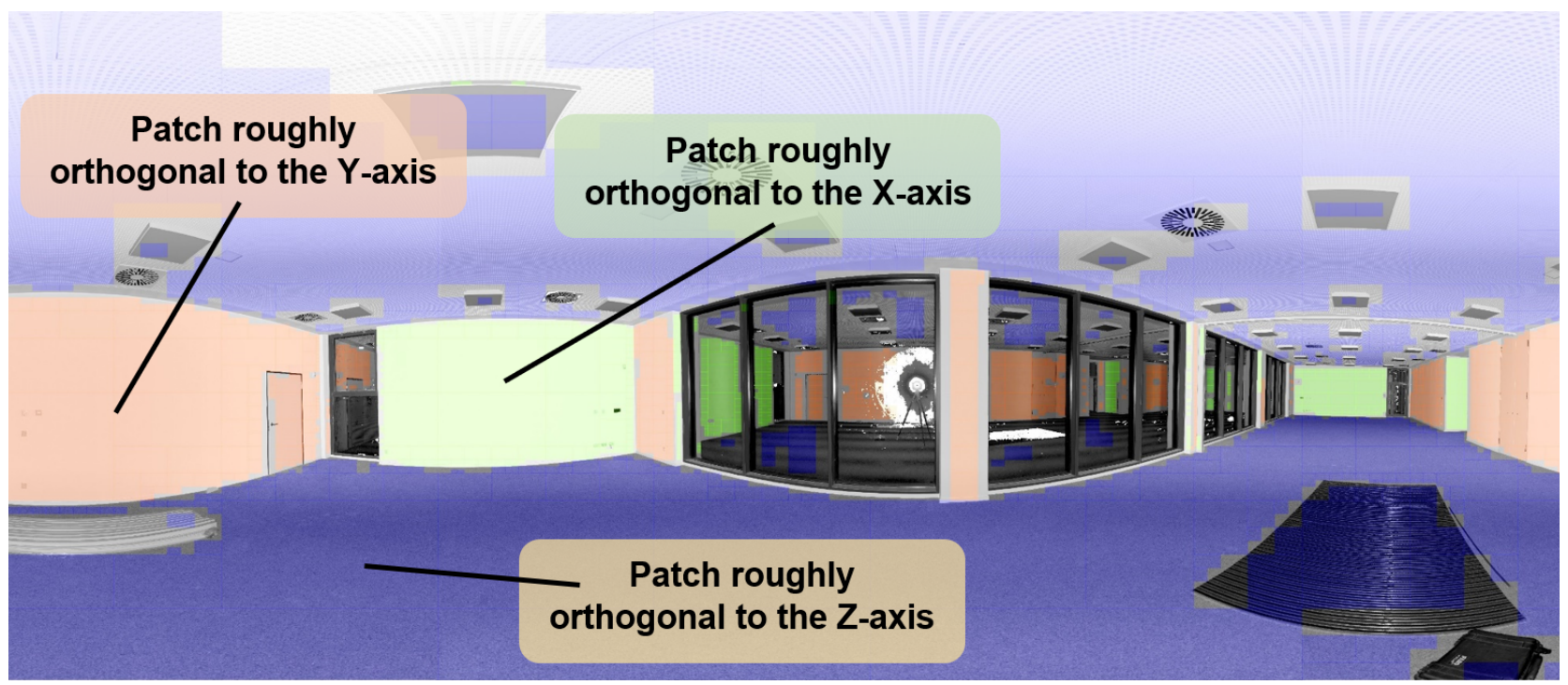

Figure 5. The detected patches are colored according to your skin direction. In our case, this also makes it easier to assign the TLS patches to the BIM faces. Please note, that the labeled patches correspondent to the faces in figures Figure 3

These parameters change the size of the found patches. Please note, that this is not relevant for co-registration, but for further analysis with the patches, e.g. construction progress monitoring.

In our approach, the internal data structure for patches and faces is identical. Only identifier, a point and a normal vector are stored. In addition, each patch has a covariance matrix that can be used to calculate the standard deviation for each point of the patch and the normal vector. Figure 5 shows the detected patches in the practical example. The three corresponding planes from the BIM model are labeled. This example shows that patches are also detected in the fixed glazing.

\subsection{Virtual station with BIM Faces}

Scantra works with a SQLlite database to store the entire information. This makes it easy to save the BIM faces as a new virtual station. Alternatively, the planes can also be inserted manually. In both cases, they can be displayed as shown in Figure 6. The BIM planes must be orthogonal to one of the (datum defining) coordinate axes. They also need an identifier and the value of the translation from the origin must be specified. This value can also be negative. Finally, it must be specified whether the normal vector of the BIM patch is positive or negative.

The virtual station is handled like a normal scan station, whereas the patches do not originated from the TLS point cloud, but from the BIM model. This means in turn, that there is no panoramic image.

\subsection{Co-registration procedure (BIM)}

After creation, the virtual station can be added to the observation-topology. The connection (edge) to at least one TLS station must be manually created. However, by means of reliability it makes sense to link more connections as the minimum configuration requires. Due to inevitable uncertainty propagation, the stations should be evenly distributed in the scanning area. After that, the procedure is the same as for TLS registration. This means that each connection to the virtual station must be considered individually and the patches in the image must be assigned to the names of the BIM planes.

\begin{tabular}{|c|c|c|c|}
\hline \multicolumn{2}{|c|}{ Virtuelle Station editieren } & \multirow[b]{2}{*}{$\begin{array}{l}\text { Named planes } \\
\text { from BIM }\end{array}$} & $x$ \\
\hline Station-ID: virtual_ & & & \\
\hline \multicolumn{2}{|l|}{ Virtuele Ebenen: } & & + \\
\hline Ebeneld & Ebene orthogonal zur & Translation NV positiv & \\
\hline 0 _green_plane & X-Achse & $0.253 \quad \square$ & \\
\hline 0_orange_plane & Y-Achse & -7.720 & \\
\hline 0_yellow_lane & Z-Achse & 0.000 & \\
\hline other_plane_1 & $x$-Achse & 5.450 & \\
\hline other_plane_2 2 & $x$-Achse & 11.680 & \\
\hline other_plane_3 3 & Y-Achse & -4.850 & \\
\hline other_plane_4 & Y-Achse & -15.800 & \\
\hline other_plane_ 5 & Y-Achse & 0.200 & \\
\hline other_plane_6 & Z-Achse & 5.200 & \\
\hline other_plane_7 & Z-Achse & 2.500 & \\
\hline \multicolumn{4}{|c|}{ Other planes, not named specifically } \\
\hline & & ok & Abbrechen \\
\hline
\end{tabular}

Figure 6. Graphical user interface for selecting the BIM faces in the registration software. If necessary, for example with layered walls, the translation can be adjusted or the orientation of the normal vector can be changed. The entire BIM is named as virtual_station for demonstration proposes.

For one connection, at least three plane pairs must be selected. The remaining plane correspondences are found automatically by the registration software. After completed matching, the coregistration of the point cloud to the virtual station system can be calculated. Then the point cloud can be transformed and imported into the BIM software for further processing. Figure 7 shows the original BIM model with the registered point cloud loaded.

After the point cloud has been successfully co-registered, it can be used for Scan vs. BIM applications or construction progress monitoring.

\section{QUALITY ASSURANCE}

The quality of the co-registration can be read from the Scantra protocol, which also allows errors to be excluded. Figure 8 shows the connections to virtual stations in a part of the protocol. The correction is particularly interesting. The total point 


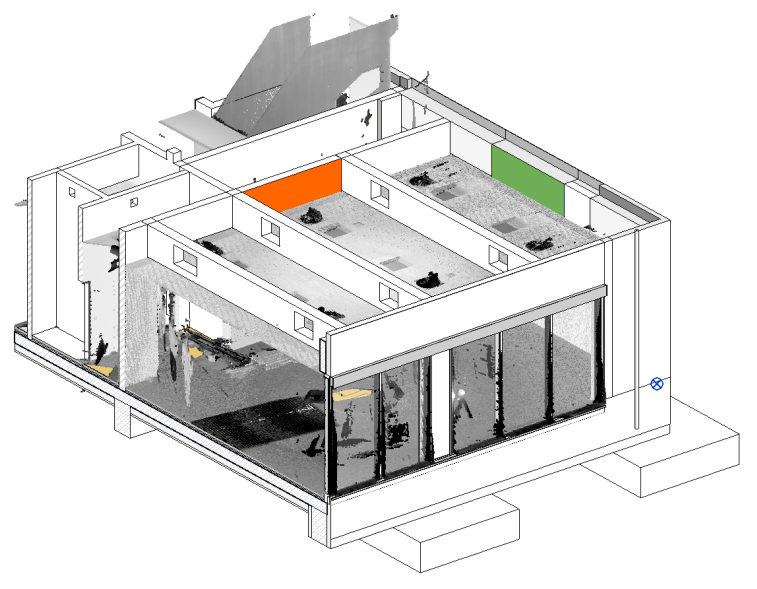

Figure 7. BIM model with finished co-registered point cloud

cloud is not distorted in itself, but positioned as best as possible between the planes. Please note that the maximum residual is approximately 2.5 centimeters which complies with the expected deviation between BIM Model and execution of construction work. If the models did not fit to the scanned reality, the co-registration would be biased. However, this would be documented in the protocol.

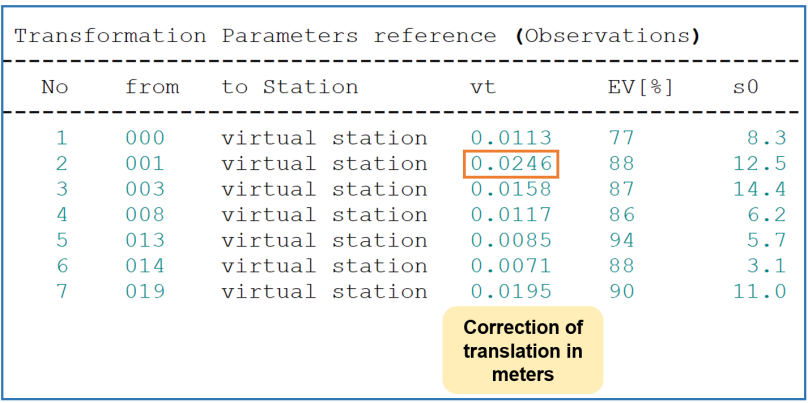

Figure 8. Section of the Scantra protocol

In the graph in Figure 9, these deviations are shown with correspondingly bold lines. The virtual station indicates the position of the BIM origin after co-registration. Additionally the protocol lists the datum-depended standard deviations for each station and the reliability as partial redundancies.

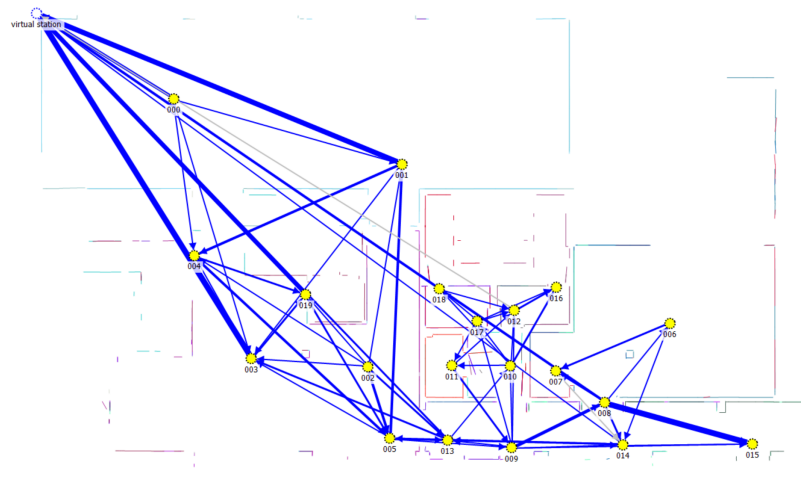

Figure 9. Result topology in Scantra with virtual station as reference

The virtual station was connected to a total of seven stations, which are well distributed in the building. This in turn means that there are no major deviations between the model and reality.

\section{CONCLUSION AND OUTLOOK}

In this contribution a patched-based co-registration with several static laser scans and BIM was demonstrated. For this purpose the software Scantra was extended. We have demonstrated how patches, extracted as BIM-faces, are assigned to TLS patches for the propose of co-registration.

For the sake of exemplification, the suggested algorithm was presented with a rather small scanning project. The registration software itself is developed for large projects with thousands of TLS stations (Wujanz et al., 2018).

The major benefit of the proceeded is that artificial control points are not required. Without co-registration, the control points would have to be installed and surveyed with additional time and effort. Planes, respectively patches, have the advantage that they occur sufficiently in high raised building structures.

The bottleneck of the procedure is the quality of the BIM. At least the datum defining faces must correspond with the captured reality. The software still has some limitations which will be considered in future works: The coordinate system in BIM currently still has to run parallel to the building axes. However, from a mathematical point of view, at least three mutually orthogonal would be sufficient for a minimal configuration.

Large stable surfaces of floor, ceilings and walls are suitable for the registration. Thus, it must also be possible to detect these in the recorded scans. In practice, care must be taken that not too many temporary materials or objects occlude the desired building components of interest.

Another advantage of plane-based registration with Scantra is that the error budget considers the precision of the scanner, its calibration and registration within a stochastic model. This means that the individual patches have an explicit standard deviation, which is typically not known for individual points of a point clouds. This information represents additional value for further work with the point clouds. Another major advantage is that the amount of data is considerably reduced without losing important information from the point cloud.

The presented approach will be used in future research on highly accurate construction progress monitoring that fulfills geodetic standards.

\section{ACKNOWLEDGEMENTS}

The research project ProgressPatch was funded by the German Federal Ministry for Economic Affairs and Energy. Grant No. $16 \mathrm{KN} 057445$.

\section{REFERENCES}

Besl, P. J., McKay, N. D., 1992. A method for registration of 3$\mathrm{D}$ shapes. IEEE Transactions on pattern analysis and machine intelligence, 239-256.

Böhm, J., Becker, S., 2007. Automatic marker-free registration of terrestrial laser scans using reflectance. In Proceedings of the 8th conference on optical 3D measurement techniques, 9-12. 
Bosché, F., 2010. Automated recognition of 3D CAD model objects in laser scans and calculation of as-built dimensions for dimensional compliance control in construction. Advanced Engineering Informatics, 24(1), 107-118. https://www.sciencedirect.com/science/article/ $\mathrm{pii} / \mathrm{S} 1474034609000482$.

Gielsdorf, F., Rietdorf, A., Gruendig, L., 2004. A Concept for the Calibration of Terrestrial Laser Scanners. Proceedings of the FIG Working Week.

Kaiser, T., Clemen, C., Maas, H.-G., 2022. Automatic coregistration of photogrammetric point clouds with digital building models. Automation in Construction, 134, 104098. https://www.sciencedirect.com/science/article/ pii/S0926580521005495.

Martens, J., Blankenbach, J., 2020. An evaluation of posenormalization algorithms for point clouds introducing a novel histogram-based approach. Advanced Engineering Informatics, 46, 101132. https://www.sciencedirect.com/science/ article/pii/S1474034620301038.

Wujanz, D., 2016. Terrestrial Laser Scanning for Geodetic Deformation Monitoring. Dissertation, Technical University of Berlin. https://dgk.badw.de/fileadmin/user_ upload/Files/DGK/docs/c-775.pdf.

Wujanz, D., Schaller, S., Gielsdorf, F., Gründig, L., 2018. Plane-based registration of several thousand laser scans on standard hardware. The International Archives of the Photogrammetry, Remote Sensing and Spatial Information Sciences, XLII-2, 1207-1212.

Zhang, C., Arditi, D., 2013. Automated progress control using laser scanning technology. Automation in Construction, 36, 108-116. https://www.sciencedirect.com/science/ article/pii/S092658051300126X. 